\title{
IDENTIFICAÇÃO DE INDICADORES CONTÁBEIS RELEVANTES PARA PREVISÃO E PROJEÇÃO DE RENTABILIDADE
}

\section{IDENTIFICATION OF RELEVANT ACCOUNTING INDICATORS FOR PROFITABILITY FORECAST AND PROJECTION}

\section{IDENTIFICACIÓN DE INDICADORES CONTABLES RELEVANTES PARA PREVISIÓN Y PROYECCIÓN DE RENTABILIDAD}

\section{FLÁVIO LEONEL DE CARVALHO}

Bacharel em Ciências Contábeis pela FEA USP; Mestre em Controladoria e Contabilidade pela FEA-RP USP; Doutorando em Engenharia de Produção pela EESC-USP, Universidade de São Paulo

flavioleocar@hotmail.com

\section{ANDREI APARECIDO DE ALBUQUERQUE}

Bacharel em Ciências Contábeis pela FEA-RP USP; MBA em Controladoria e Finanças pela Fundace/USP; Mestre em Controladoria e Contabilidade pela FEA-RP USP; Pesquisador da FEA-RP USP; Professor da Universidade Federal de Uberlândia andreialbuq@yahoo.com

\section{RAPHAEL PAZZETTO GONÇALVES}

Bacharel em Ciências Econômicas FEA-RP US; Mestre em Controladoria e Contabilidade pela FEA-RP USP; Pesquisador da FEA-RP USP

Raphael_pg@yahoo.com.br.

\section{MARLI AUXILIADORA DA SILVA}

Bacharel em Ciências Contábeis; Especialista em Gestão de Pequenos Negócios pela UFLA MG; Mestre em Controladoria e Contabilidade pela FEA-RP USP; Pesquisadora da FEA-RP USP; Professora da Universidade Federal de Uberlândia 


\title{
EVANDRO MARCOS SAIDEL RIBEIRO
}

Bacharel, Mestre e Doutor em Física pela UFSCar com Pós-Doutorado em Física pela UNICAMP e IFSC-USP; Professor doutor e membro titular na comissão de pesquisa da FEA-RP da USP-Universidade de São Paulo, Faculdade de Economia, Administração e

Contabilidade de Ribeirão Preto

saidel@fearp.usp.br

\section{RESUMO}

Vários estudos utilizando indicadores contábeis para a previsão de insolvência têm sido desenvolvidos desde as décadas de sessenta e setenta. Esses trabalhos buscam prever um possível estado de deterioração do desempenho financeiro das empresas, utilizando-se de modelos estatísticos. A presente pesquisa buscou identificar quais os indicadores contábeis mais significativos para a previsão de rentabilidade das empresas, propondo uma função que utilize indicadores passados como base para prever rentabilidade futura. Foram empregadas informações de todas as empresas não financeiras que estavam ativas e possuíam ações negociadas na Bolsa de Valores de São Paulo (Bovespa) nos anos de 2004, 2003 e 2002. Utilizando-se a análise descritiva e empregando o modelo de regressão linear múltipla, verificaram-se quais foram as variáveis mais significativas com suas respectivas ponderações. Conclui-se com uma função de previsão de ROA, contendo cinco variáveis independentes estatisticamente significantes ao nível de $5 \%$ (cinco porcento) das doze selecionadas para o modelo inicial.

Palavras-chave: Indicadores Contábeis. Rentabilidade das Empresas. Regressão Múltipla.

\begin{abstract}
Several studies using accounting indicators for insolvency prediction have been developed since 1960s and 1970s. These works seek to predict a possible deterioration status of companies' financial performance by using statistical models. This survey sought to identify which are the most significant accounting indicators for profitability prediction of firms, proposing a function that uses past indicators as basis to predict future profitability. It was used information from all non-financial companies that were active and had stocks traded in Sao Paulo Stock Exchange (Bovespa) in 2004, 2003 and 2002. Using descriptive analysis and applying multiple linear regression model, it was verified which were the most significant variables with their respective relevance. It was completed with a ROA prediction function, containing five independent variables statistically significant at $5 \%$ (five percent) level, out of twelve selected for the initial model.
\end{abstract}

Key words: Accounting Indicators; Companies' Profitability; Multiple Regression 


\section{repec}

Flávio Leonel de Carvalho, Andrei Aparecido de Albuquerque, Raphael Pazzetto Gonçalves, Marli Auxiliadora da Silva e Evandro Marcos Saidel Ribeiro

\section{RESUMEN}

Varios estudios utilizando indicadores contables para la previsión de insolvencia han sido desarrollados desde las décadas de sesenta y setenta. Esos trabajos buscan prever un posible estado de deterioración del desempeño financiero de las empresas, haciendo uso de modelos estadísticos. La presente pesquisa buscó identificar cuáles son los indicadores contables más significativos para la previsión de rentabilidad de las empresas, proponiendo una función que utilice indicadores pasados como base para prever rentabilidad futura. Fueron empleadas informaciones de todas las empresas no financieras que estaban activas y poseían acciones negociadas en la Bolsa de Valores de São Paulo (Bovespa) en los años 2004, 2003 y 2002. Haciendo uso del análisis descriptivo y empleando el modelo de regresión lineal múltiple, se verificaron cuales fueron las variables más significativas con sus respectivas ponderaciones. Se concluye con una función de previsión de ROA, conteniendo cinco variables independientes estadísticamente significantes al nivel del 5\% (cinco por ciento) de las doce seleccionadas para el modelo inicial.

Palabras clave: Indicadores Contables. Rentabilidad de las Empresas. Regresión Múltiple.

\section{INTRODUÇÃO}

Uma questão constante quando se discute a avaliação de uma empresa é a validade das informações fornecidas pela contabilidade. Muitos autores defendem que, apesar de os sistemas de informações contábeis serem a principal fonte de dados contábeis, financeiros e econômicos das empresas, essas informações não são confiáveis. Por outro lado, há alguns estudos que comprovam empiricamente que os índices contábeis, ao contrário do que se pensa popularmente, fornecem informações antecipadas valiosas sobre o processo de deterioração da situação financeira das entidades de um modo geral (SANVICENTE; MINARDI, 1998; GIMENES; URIBE-OPAZO, 2006).

Partindo da premissa de que as informações contábeis são confiáveis e fornecem informações valiosas, o presente trabalho buscou verificar se é possível prever o desempenho das empresas antecipadamente (medido por meio dos índices de retorno sobre o patrimônio líquido - ROE - e retorno sobre os ativos - ROA), tendo, por conseguinte o objetivo de identificar os indicadores contábeis mais relevantes para o processo de projeção e previsão da rentabilidade.

A projeção de rentabilidade é um dos objetivos almejados quando são feitas análises dos demonstrativos contábeis. Se a projeção for realizada de maneira adequada e coerente poderão ser reduzidos os riscos de decisões inadequadas e medidas corretivas serão tomadas quando uma situação de ineficiência for detectada, tendo como meta corrigir e redirecionar a situação econômico-financeira das entidades.

O presente artigo teve um caráter empírico e, buscando atingir o objetivo proposto, qual seja identificar quais índices contábeis são mais significativos para a previsão de ren- 
tabilidade das empresas no Brasil, utilizou-se a regressão múltipla para esse fim. Adotou-se como medida de rentabilidade (variáveis dependentes) o retorno sobre os ativos (ROA) e o retorno sobre patrimônio líquido (ROE) do ano de 2005 ( $t$ ), sendo utilizados alguns dos tradicionais índices contábeis (índices de eficiência financeira, liquidez, endividamento, imobilização de ativos, eficiência econômica, etc.) como variáveis independentes com um, dois e três anos de defasagem ((t-1), (t-2) e (t-3)) em relação ao ROA e ROE do ano $t$ (2005). Assim, tiveram-se como variáveis dependentes o ROA e o ROE de 2005 e como variáveis independentes os indicadores contábeis de 2004, 2003 e 2002, representados abaixo por $X_{i}, i=1,2, \ldots, 12$, para os anos de $(t-1),(t-2)$ e $(t-3)$.

As relações estudadas foram, portanto:

$$
\begin{aligned}
& R O E_{(t)}=\alpha_{R O E}+\sum_{i=1}^{\mathcal{L}} \beta_{i} \mathrm{X}_{i}(t-p) p=1,2 e 3 \\
& R O A_{(t)}=\alpha_{R O A}+\sum_{i=1}^{\mathcal{L}} \sigma_{i} \mathrm{X}_{i}(t-p) p=1,2 e 3
\end{aligned}
$$

Onde, $t$ foi o ano base do estudo, ou seja, 2005 e $p$ o fator de defasagem do ano das variáveis independentes, portanto, 1, 2 e 3, resultando nos anos de 2004, 2003 e 2002, o $\alpha$ refere-se à intersecção e $\beta$ ao coeficiente angular de cada uma das variáveis.

Por meio da regressão, pôde-se identificar quais indicadores (variáveis independentes) apresentam uma relação linear com o ROA e ROE, determinando, dessa forma, a importância de cada um deles e seu respectivo peso na determinação do modelo.

\section{REVISÃO BIBLIOGRÁFICA}

Desde o seu surgimento, a Contabilidade tem servido como instrumento para controle e auxílio ao processo de decisão, sendo que seu objetivo inicial era produzir informações somente para os proprietários das empresas. Ao longo da história, surgiram novos interessados no acompanhamento e avaliação do desempenho das empresas. Desse modo, o objeto da Contabilidade teve que se readequar a essa nova realidade (HENDRIKSEN; VAN BREDA, 1999). Consoante Casa Nova (2002), desde o seu surgimento, durante o Renascimento, na região de Veneza, a Contabilidade tem servido para a análise de desempenho de empresas.

Em 1919, Wall desenvolve um modelo de análise das demonstrações contábeis por meio de índices, no intuito de avaliar e desenvolver técnicas eficazes de monitoramento da gestão das empresas (MATARAZZO, 1995). Para o mesmo autor, o estudo desenvolvido por Wall foi a primeira tentativa de atribuição de pesos, no qual se ponderaram os vários índices para se alcançar a "Fórmula de Wall". Por meio da relação entre contas contábeis, a metodologia adotada reúne alguns índices e parâmetros para análise de balanços e para a avaliação de desempenho financeiro de empresas. 


\section{repec}

Flávio Leonel de Carvalho, Andrei Aparecido de Albuquerque, Raphael Pazzetto Gonçalves, Marli Auxiliadora da Silva e Evandro Marcos Saidel Ribeiro

Conforme Silva (1997), o pesquisador Fitz Patrick, além de realizar a análise das demonstrações financeiras por meio de índices de relacionamento entre contas, promove, em 1932, um estudo no qual seleciona aleatoriamente 19 empresas que haviam falido no período de 1920 a 1929 e as compara com outras 19 empresas bem-sucedidas, sendo, portanto, o pioneiro na elaboração de modelos estatísticos de previsão de insolvência.

Em 1966, Beaver, utilizando-se de índices contábeis, propõe um modelo de previsão de dificuldade financeira. Com isso, o autor procura elucidar os seguintes pontos: (a) quais eram os indicadores contábeis mais relevantes para a previsão de dificuldades financeiras; (b) quão eficientes eram esses indicadores; e (c) a partir de uma determinada situação financeira avaliada pelos índices contábeis, qual a probabilidade de uma empresa falir.

Dois anos após a realização do estudo de Beaver (1966), Altman (1968) iniciou um trabalho sobre a falência de empresas no qual correlacionou diversos indicadores contábeis. Com essa análise conjunta dos índices, ele demonstrou que a falência de uma companhia poderia ser prevista pelas informações disponíveis nos balanços patrimoniais.

Os trabalhos relatados anteriormente são tidos como marcos para a pesquisa sobre previsão de dificuldades financeiras, falência e insolvência de empresas, sendo que até hoje inúmeros outros artigos foram desenvolvidos no sentido de contribuir com os primeiros resultados alcançados.

Verifica-se também que, nos últimos anos, várias pesquisas foram realizadas utilizando diferentes ferramentas estatísticas, tendo como principal objetivo a previsão de falência e insolvência de empresas.

No Brasil, Kanitz (1976) desenvolveu um modelo de previsão no qual emprega análise discriminante para estimar previsão de insolvência de empresas. Para realizar seu trabalho, o autor utilizou aproximadamente cinco mil balanços de empresas participantes do estudo da revista "Exame Maiores e Melhores", sendo que, utilizando essas informações, ele desenvolve o indicador de insolvência conhecido como "Termômetro de Insolvência de Kanitz", o qual apresenta escores que variam de $-7 \mathrm{a}+7$.

Gimenes e Uribe-Opazo (2001), utilizando-se de técnicas estatísticas multivariadas, especialmente $a$ análise discriminante e a análise de probabilidade condicional, desenvolvem um trabalho com o objetivo de provar, com evidências empíricas, que os demonstrativos contábeis fornecem informações valiosas sobre o processo de deterioração dos índices financeiros. Os autores se utilizam de informações de cooperativas agropecuárias para provar suas hipóteses e concluem que os demonstrativos contábeis dessas entidades podem fornecer informações importantes e seguras para se antecipar situações de desequilíbrio financeiro.

\section{1 Índices financeiros e avaliação de desempenho}

Segundo Catelli (2001, p. 198), avaliação refere-se ao ato ou efeito de se atribuir valor, sendo que pode ser entendido em um sentido qualitativo (mérito ou importância) 


\section{repec}

ou em um sentido quantitativo (mensuração), sendo que a mensuração do desempenho, no sentido quantitativo, utilizaria de indicadores numéricos como: percentuais, quocientes, montantes, multiplicadores, como forma de aferição (CASA NOVA, 2002, p. 35).

De acordo com Matarazzo (1998), a avaliação de empresas por meio de índices exige, obrigatoriamente, a comparação com padrões e, dessa forma, é importante que haja a fixação da importância relativa de cada índice.

Abe e Fama (1999) ratificam o quanto é importante a utilização de índices no processo de análise do desempenho financeiro de empresas, enquanto Matarazzo (1995, p. 153) afirma que este índice "[...] é a relação entre contas ou grupos de contas das demonstrações financeiras que visa evidenciar determinado aspecto da situação econômica ou financeira de uma empresa". Dessa forma, os indicadores constituem a metodologia de análise mais empregada e tem como objetivo fornecer uma visão ampla da situação econômicofinanceira das empresas.

\section{METODOLOGIA}

Nessa seção, são descritos os principais aspectos metodológicos da pesquisa.

\subsection{Objetivo geral da pesquisa}

Este estudo teve por objetivo identificar quais são os indicadores contábeis mais significativos para a previsão de rentabilidade das empresas, propondo uma função que utilize indicadores passados como base para prever rentabilidade futura.

Para a consecução de tal objetivo, este estudo fez (a) uma aplicação da técnica estatística de regressão linear múltipla para projeção de rentabilidade; (b) selecionou os indicadores a serem utilizados na avaliação de desempenho medido por meio da rentabilidade; (c) identificou a importância de avaliações por meio de indicadores financeiros de forma integrada; e (d) estruturou uma função linear para a previsão e projeção da rentabilidade.

\subsection{Caracterização da pesquisa e seleção da amostra}

Esta pesquisa é de natureza quantitativa e exploratória, sendo que os dados contábeis utilizados são caracterizados como secundários, uma vez que foram obtidos da base de dados da Economática ${ }^{\circledR}$.

Inicialmente, foram extraídas informações de todas as empresas não financeiras que estavam ativas e possuíam ações negociadas na Bolsa de Valores de São Paulo (Bovespa) em 2004, 2003 e 2002. Em seguida, foram selecionadas as contas contábeis necessárias para calcular os indicadores. As empresas que possuíam dados nulos em qualquer uma das informações necessárias foram excluídas da amostra. Do total de informações obtidas, foram selecionadas 100, 101 e 110 empresas para os anos de 2002, 2003 e 2004, respectivamente. 


\section{repec}

Flávio Leonel de Carvalho, Andrei Aparecido de Albuquerque, Raphael Pazzetto Gonçalves, Marli Auxiliadora da Silva e Evandro Marcos Saidel Ribeiro

\section{DESCRIÇÃO DA PESQUISA}

Baseando-se nas informações extraídas pela Economática ${ }^{\circledR}$, calcularam-se 12 indicadores contábeis, que representaram as variáveis independentes. Os indicadores utilizados foram:

- $\mathrm{X}_{1}$ : (ativo circulante - passivo total) / ativo total

- $\mathrm{X}_{2}$ : (patrimônio líquido - capital social) / ativo total

- $\mathrm{X}_{3}$ : (lucro operacional - despesas financeiras + receitas financeiras) / ativo total

- $\mathrm{X}_{4}$ : valor contábil do patrimônio líquido / valor contábil do exigível total

- $\mathrm{X}_{5}$ : receita líquida / ativo total

- $\mathrm{X}_{6}$ : (ativo circulante operacional - passivo circulante operacional) / ativo total

- $\mathrm{X}_{7}$ : (ativo circulante financeiro - passivo circulante financeiro) / ativo total

- $\mathrm{X}_{8}$ : lucro operacional antes de juros e imposto de renda / despesas financeiras

- $\quad X_{g}$ : fluxo de caixa das operações $/$ ativo total $=($ LAJIR + depreciação - investimento em capital de giro) / ativo total

- $\mathrm{X}_{10}$ : investimento permanente / ativo total

- $\mathrm{X}_{11}$ : impostos / ativo total

- $\mathrm{X}_{12}$ : variação do disponível / ativo total

As variáveis $X_{1}$ a $X_{5}$ foram idênticas às utilizadas por Sanvicente e Minardi (1998) e, consoante esses autores, as cinco primeiras variáveis foram testadas no Brasil por Altman, Baidya e Dias, em 1977, e por Sanvicente e Bader, em 1996. Já as variáveis $\mathrm{X}_{6}$ e $\mathrm{X}_{7}$ referem-se a medidas de liquidez que controlam o fenômeno de overtrading citado por Assaf Neto e Silva (2002). A variável X8 corresponde ao índice de cobertura de juros, que conforme Sanvicente e Minardi (1998) é uma das variáveis consideradas na avaliação de ratings pela agência Standard \& Poor's e as variáveis $\mathrm{X}_{9}$ a $\mathrm{X}_{12}$, de acordo com os supracitados autores foram sugeridas por Mossman, Bell, Swartz e Turtle (1998) em um modelo discriminante baseado em dados de fluxo de caixa.

Como variáveis dependentes foram usadas as seguintes variáveis:

- $Y_{1}: R O A=$ (lucro líquido $/$ ativo total médio)

- $Y_{2}: R O E=$ (lucro líquido $/$ patrimônio líquido médio)

Utilizou-se a ferramenta estatística regressão múltipla para identificação dos indicadores contábeis que explicavam as variações no ROE e no ROA. Inicialmente, rodou-se a regressão considerando a variável $R O E_{2005}$ como variável dependente e as variáveis $X_{1}$ a $X_{12}$ de 2004, 2003 e 2002 como independentes. Posteriormente, o mesmo procedimento foi adotado, alterando-se apenas a variável dependente que passou a ser o $\mathrm{ROA}_{2005}$. 


\section{repec}

\subsection{Descrição dos procedimentos de tratamento dos dados}

Foram rodadas seis regressões, sendo três para testar se é possível prever o ROE de 2005 utilizando indicadores contábeis de 2004, 2003 e 2002, e as outras três avaliando a previsibilidade do ROA, também de 2005 , em relação às mesmas variáveis independentes, ou seja, índices contábeis dos três anos em análise.

Em geral, testaram-se as hipóteses

$$
\begin{aligned}
& H_{0}^{R O E}: \beta_{i}=0 \mathrm{e} \\
& H_{0}^{R O A}: \sigma_{i}=0
\end{aligned}
$$

que podem ser detalhadas e apresentadas de forma mais simplificada como segue:

$\mathrm{H}_{1}$ : Existe relação linear entre as variáveis $\left(\mathrm{X}_{1}\right.$ à $\left.\mathrm{X}_{12}\right)$ de 2004 e ROE de 2005.

$\mathrm{H}_{2}$ : Existe relação linear entre as variáveis $\left(\mathrm{X}_{1}\right.$ à $\left.\mathrm{X}_{12}\right)$ de 2003 e ROE de 2005.

$\mathrm{H}_{3}$ : Existe relação linear entre as variáveis $\left(\mathrm{X}_{1}\right.$ à $\left.\mathrm{X}_{12}\right)$ de 2002 e ROE de 2005.

$\mathrm{H}_{4}$ : Existe relação linear entre as variáveis $\left(\mathrm{X}_{1}\right.$ à $\left.\mathrm{X}_{12}\right)$ de 2004 e ROA de 2005.

$H_{5}$ : Existe relação linear entre as variáveis $\left(X_{1}\right.$ à $\left.X_{12}\right)$ de 2003 e ROA de 2005.

$\mathrm{H}_{6}$ : Existe relação linear entre as variáveis $\left(\mathrm{X}_{1}\right.$ à $\left.\mathrm{X}_{12}\right)$ de 2002 e ROA de 2005.

\subsubsection{Previsão de ROE}

Nesta etapa do trabalho, verificou-se a possibilidade de previsão do ROE por meio dos índices contábeis defasados em um, dois ou três anos. O modelo é descrito pela expressão (1):

$$
H_{1}^{R O E}: \beta_{i} \neq 0
$$

Ou seja,

$\mathrm{H}_{1}: \operatorname{ROE}(2005)=\alpha+\beta \mathrm{X}_{1}(2004)+\beta \mathrm{X}_{2}(2004)+\ldots+\beta \mathrm{X}_{11}(2004)+\beta \mathrm{X}_{12}(2004)+\varepsilon$

$\mathrm{H}_{2}: \operatorname{ROE}(2005)=\alpha+\beta \mathrm{X}_{1}(2003)+\beta \mathrm{X}_{2}(2003)+\ldots+\beta \mathrm{X}_{11}(2003)+\beta \mathrm{X}_{12}(2003)+\varepsilon$

$\mathrm{H}_{3}: \operatorname{ROE}(2005)=\alpha+\beta \mathrm{X}_{1}(2002) \beta \mathrm{X} 2(2002)+\ldots+\beta \mathrm{X}_{11}(2002)+\beta \mathrm{X}_{12}(2002)+\varepsilon$

Os coeficientes de determinação das regressões utilizadas para previsão do ROE apresentaram grau de ajustamento com a reta baixo nos três anos $(0,0795,0,0533$ e 0,1086$)$. Como pode ser observado na Tabela 1, em todos os anos bases do estudo, esse valor ficou abaixo de $11 \%$, o que indica que menos de $11 \%$ das variações no ROE podem ser explicadas pelas alterações nas variáveis independentes. Além disso, o valor $F$ de significação é maior que $5 \%$ nos três anos, ou seja, não se rejeita a hipótese nula de que não existe relação linear entre as variáveis dependentes e independentes das hipóteses 1 a $3\left(H_{0}^{R O E}: \beta_{i}=0 ; \mathrm{i}=1,2\right.$ e 3). 


\section{repec}

Flávio Leonel de Carvalho, Andrei Aparecido de Albuquerque, Raphael Pazzetto Gonçalves, Marli Auxiliadora da Silva e Evandro Marcos Saidel Ribeiro

Tabela 1 - Coeficiente de determinação e $F$ de significação dos anos 2002 a 2004 para previsão de ROE

\begin{tabular}{lccc}
\hline & $\mathbf{2 0 0 2}$ & $\mathbf{2 0 0 3}$ & $\mathbf{2 0 0 4}$ \\
\hline$R$-quadrado ajustado & $(0,0795)$ & $(0,0533)$ & $(0,1086)$ \\
F de significação & 0,9629 & 0,8542 & 0,9999 \\
Observações & 100 & 101 & 110 \\
\hline
\end{tabular}

Na Tabela 2, estão descritos os valores $p$ para todas as variáveis independentes em análise e nota-se que todas são superiores a $5 \%$, o que impede a afirmação de que existe um relacionamento linear significativo a esse nível entre a variável ROE e as demais variáveis independentes. Conclui-se, portanto, que não é possível afirmar que exista um relacionamento entre as variáveis independentes defasadas $X_{1}$ a $X_{12}$ e ROE.

Tabela 2 - Valor $p$ para as variáveis independentes dos anos 2002 a 2003 para previsão de ROE

\begin{tabular}{lccc}
\hline & $\begin{array}{c}\mathbf{2 0 0 2} \\
\text { valor } \boldsymbol{p}\end{array}$ & $\begin{array}{c}\mathbf{2 0 0 3} \\
\text { valor } \boldsymbol{p}\end{array}$ & $\begin{array}{c}\mathbf{2 0 0 4} \\
\text { valor } \boldsymbol{p}\end{array}$ \\
\hline $\mathrm{X}_{1}$ & 0,8277 & 0,9885 & 0,9953 \\
$\mathrm{X}_{2}$ & 0,9515 & 0,3285 & 0,7441 \\
$\mathrm{X}_{3}$ & 0,2255 & 0,0791 & 0,7636 \\
$\mathrm{X}_{4}$ & 0,9326 & 0,8750 & 0,9705 \\
$\mathrm{X}_{5}$ & 0,9247 & 0,2524 & 0,9851 \\
$\mathrm{X}_{6}$ & 0,7212 & 0,3322 & 0,8690 \\
$\mathrm{X}_{7}$ & 0,8213 & 0,7373 & 0,6267 \\
$\mathrm{X}_{8}$ & 0,7482 & 0,8560 & 0,8105 \\
$\mathrm{X}_{9}$ & 0,8347 & 0,8058 & 0,6194 \\
$\mathrm{X}_{10}$ & 0,3189 & 0,1598 & 0,9195 \\
$\mathrm{X}_{11}$ & 0,9092 & 0,2445 & 0,7634 \\
$\mathrm{X}_{12}$ & 0,7110 & 0,4677 & 0,8405 \\
\hline
\end{tabular}

\subsubsection{Previsão de ROA}

O procedimento descrito anteriormente foi repetido para a variável dependente ROAe, nessa etapa do trabalho, foram testadas as hipóteses de que é possível prever ROA por meio dos índices contábeis de um, dois e três anos de defasagem. Sendo o modelo descrito pela expressão

$$
H_{0}^{R O A}: \sigma_{i} \neq 0 ; \mathrm{i}=1,2 \text { e } 3
$$

que pode ser detalhada em

$$
\begin{aligned}
& \mathrm{H}_{4}: \mathrm{ROA}_{2005}=\alpha+\sigma \mathrm{X}_{1(2004)}+\sigma \mathrm{X}_{2(2004)}+\ldots+\sigma \mathrm{X}_{11(2004)}+\sigma \mathrm{X}_{12(2004)}+\varepsilon \\
& \mathrm{H}_{5}: \mathrm{ROA}_{2005}=\alpha+\sigma \mathrm{XI}_{1(2003)}+\sigma \mathrm{X}_{2(2003)}+\ldots+\sigma \mathrm{X}_{1(2003)}+\sigma \mathrm{X}_{12(2003)}+\varepsilon \\
& \mathrm{H}_{6}: \mathrm{ROA}_{2005}=\alpha+\sigma \mathrm{X}_{1(2002)}+\sigma \mathrm{X}_{2(2002)}+\ldots+\sigma \mathrm{X}_{11(2002)}+\sigma \mathrm{X}_{12(202)}+\varepsilon
\end{aligned}
$$


O estudo do ROA foi desenvolvido em três etapas. Inicialmente utilizou-se o mesmo procedimento adotado para o ROE. Como o modelo apresentou um poder de explicação significativo em todos os anos (como pode ser verificado na Tabela 3 pelos valores dos $R^{2}$ ajustados), foi rodada uma nova regressão, excluindo-se as variáveis independentes que, individualmente, apresentavam um valor $P$ maior do que $5 \%$. Após a exclusão das variáveis, novamente o resultado obtido apresentou uma variável independente com valor $P$ acima de $5 \%$ e, devido a esse resultado, todo o procedimento descrito anteriormente foi repetido, resultando em uma função com apenas cinco variáveis explicativas.

\subsubsection{Previsão do ROA - Etapa 1}

A Tabela 3 mostra que as regressões que comparam a rentabilidade dos ativos (ROA) com os indicadores obtiveram resultado significativo.

Tabela 3 - Coeficiente de determinação e F de significação dos anos 2002 a 2004 para previsão de ROA

\begin{tabular}{|c|c|c|c|}
\hline & 2002 & 2003 & 2004 \\
\hline$R$-quadrado ajustado & 0,5483 & 0,9946 & 0,8339 \\
\hline F de significação & 0,0000 & 0,0000 & 0,0000 \\
\hline Observações & 100 & 101 & 110 \\
\hline
\end{tabular}

Nota-se que os $R^{2}$ ajustados para os três anos apresentam um nível elevado de ajustamento à reta. Aproximadamente $55 \%$ das variações no $\mathrm{ROA}_{2005}$ são explicadas pelas variáveis independentes $X_{1}$ a $X_{12}$ de 2002. Além disso, as variações de $X_{1}$ a $X_{12}$ de 2003 e 2004 explicam, respectivamente, 99,46\% e 83,39\% das variações de $\mathrm{ROA}_{2005}$. Ao mesmo tempo, o $F$ de significação é aproximadamente zero nos três anos. Diante desses dados, podem-se rejeitar as hipóteses nulas para as hipóteses $\mathrm{H}_{4}, \mathrm{H}_{5}$ e $\mathrm{H}_{6}$.

Tabela 4 - Coeficientes e valores $p$ das variáveis independentes - ROA

\begin{tabular}{lcccccc}
\hline & \multicolumn{2}{c}{2002} & \multicolumn{2}{c}{2003} & \multicolumn{2}{c}{2004} \\
\hline & Coeficientes & Valor $p$ & Coeficientes & Valor $p$ & Coeficientes & Valor $p$ \\
Interseção & $-1,5588$ & 0,0000 & $-0,2200$ & 0,0965 & $-0,1001$ & 0,1056 \\
$X_{1}$ & $-0,0827$ & 0,5831 & 0,1063 & 0,0031 & $-0,1094$ & 0,0000 \\
\hline$X_{2}$ & $-0,1265$ & 0,2353 & $-0,0204$ & 0,5068 & 0,0304 & 0,0000 \\
\hline$X_{3}$ & 0,5826 & 0,0348 & 1,0271 & 0,0000 & 0,5904 & 0,0000 \\
\hline$X_{4}$ & 0,0652 & 0,1175 & $-0,0221$ & 0,1018 & 0,0030 & 0,0623 \\
\hline$X_{5}$ & 0,2073 & 0,0019 & 0,0102 & 0,8003 & $-0,0517$ & 0,1003 \\
\hline$X_{6}$ & 1,8574 & 0,0000 & $-0,0089$ & 0,9601 & 0,2941 & 0,0089 \\
\hline$X_{7}$ & 0,5378 & 0,0159 & $-0,2668$ & 0,0060 & 0,2577 & 0,0033 \\
\hline
\end{tabular}




\begin{tabular}{lllllll}
\hline $\mathrm{X}_{8}$ & 0,0012 & 0,3419 & $-0,0001$ & 0,9060 & $-0,0013$ & 0,5725 \\
\hline $\mathrm{X}_{9}$ & 0,3695 & 0,0126 & $-0,2223$ & 0,0000 & 0,0756 & 0,4039 \\
\hline $\mathrm{X}_{10}$ & 1,6655 & 0,0000 & 0,3175 & 0,0269 & 0,0241 & 0,7367 \\
\hline $\mathrm{X}_{11}$ & 0,0600 & 0,7312 & 0,0274 & 0,8234 & 0,2361 & 0,0307 \\
\hline $\mathrm{X}_{12}$ & 0,8181 & 0,0484 & $-0,3561$ & 0,2448 & 0,1150 & 0,3373 \\
\hline
\end{tabular}

Pela análise dos valores $P$, disponíveis na Tabela 4, observa-se que, para os três anos, existe um relacionamento linear entre as variáveis independentes individualmente $e$ o ROA, com exceção das variáveis $X_{1}, X_{2}, X_{4}, X_{8}$ e $X_{11}$ para $2002, X_{2}, X_{4}, X_{5}, X_{6}, X_{8}, X_{11}$ e $X_{12}$ para 2003 e $X_{4}, X_{5}, X_{8}, X_{9}, X_{10}$ e $X_{12}$ para 2004, que, quando analisadas individualmente, apresentaram valor $\mathrm{P}$ acima de $5 \%$, o que impossibilita afirmar que existe relacionamento linear entre essas variáveis independentes individualmente e entre o ROA.

Para que a regressão e, consequentemente, os testes estatísticos apresentados anteriormente tenham validade plena, de acordo com Corrar e Theóphilo (2004), é necessário que sejam obedecidos os pressupostos básicos da regressão, quais sejam: a independência de erros ou correlação residual; a homocedasticidade; e a necessidade de distribuição normal dos resíduos. Para testar esses pressupostos, foram utilizados os testes de DurbinWatson, de Pesaran-Pesaran e Kolmogorov-Smirnov, e os resultados são apresentados nas Tabelas 5, 6 e 7.

Para que a regressão e, consequentemente, os testes estatísticos apresentados anteriormente tenham validade plena, de acordo com Corrar e Theóphilo (2004), é necessário que sejam obedecidos os pressupostos básicos da regressão, quais sejam: a independência de erros ou correlação residual; a homocedasticidade; e a necessidade de distribuição normal dos resíduos. Para testar esses pressupostos, foram utilizados os testes de DurbinWatson, de Pesaran-Pesaran e Kolmogorov-Smirnov, e os resultados são apresentados nas Tabelas 5, 6 e 7.

Tabela 5 - Teste Durbin-Watson - Presença significativa de autocorrelação

\begin{tabular}{ccc}
\hline & \multicolumn{2}{c}{ Teste de Durbin-Watson } \\
\hline Ano & DW & Conclusão \\
\hline 2002 & 2,2377 & Região não conclusiva \\
2003 & 2,2293 & Região não conclusiva \\
2004 & 2,0532 & Ausência de autocorrelação \\
\hline
\end{tabular}

Analisando-se a distribuição dos resíduos por meio do teste de Pesaran-Pesaran (Tabela 6), observa-se que, para o ano de 2002, não é possível rejeitar a hipótese de distribuição heterocesdástica dos resíduos. Por outro lado, para os anos de 2003 e 2004, que apresentaram valor $P$ elevado, pode-se concluir que os resíduos são homocedásticos. 
Tabela 6 - Teste Pesaran-Pesaran - Variância dos resíduos

\begin{tabular}{ccc}
\hline & \multicolumn{2}{c}{ Teste de Durbin-Watson } \\
\hline Ano & DW & Conclusão \\
\hline 2002 & 2,2377 & Região não conclusiva \\
2003 & 2,2293 & Região não conclusiva \\
2004 & 2,0532 & Ausência de autocorrelação \\
\hline
\end{tabular}

Finalmente, na Tabela 7, verifica-se que a normalidade é encontrada na distribuição dos três anos da análise.

Tabela 7 - Teste Kolmogorov-Smirnov - Distribuição dos resíduos

\begin{tabular}{cccc}
\hline & \multicolumn{2}{c}{ Teste de Kolmogorov-Smirnov } \\
\hline Ano & D teste & D crítico & Conclusão \\
\hline 2002 & 0,0127 & 0,1360 & Normalidade dos resíduos \\
2003 & 0,0002 & 0,1353 & Normalidade dos resíduos \\
2004 & 0,0214 & 0,1297 & Normalidade dos resíduos \\
\hline
\end{tabular}

Como pode ser observado anteriormente, o único ano em que todos os pressupostos da regressão são atendidos é 2004. Nesse ano, portanto, é possível a afirmativa de previsibilidade do ROA com um ano de antecedência, ou seja, a hipótese de que existe relação linear entre as variáveis independentes, de 2004, e ROA, de $2005\left(\mathrm{H}_{4}\right)$, não é rejeitada, utilizando-se a seguinte função:

\footnotetext{
$\mathrm{ROA}=-0,1001-0,1094 * \mathrm{X}_{1}(2004)+0,0304 * \mathrm{X}_{2}(2004)+0,5904 * \mathrm{X}_{3}(2004)+0,003 * \mathrm{X}_{4}(2004)-$ $0,0517 * X_{5}(2004)+0,2941 * X_{6}(2004)+0,2577 * X_{7}(2004)-0,0013 * X_{8}(2004)+0,0756 * X_{9}(2004)+0,0241$ ${ }^{*} X_{10}(2004)+0,2361 * X_{11}(2004)+0,115 * X_{12}(2004)$
}

\subsubsection{Previsão do ROA - Etapa 2}

$\mathrm{Na}$ etapa anterior, o modelo como um todo foi validado pelo $F$ de significação e o $R^{2}$ ajustado tem um razoável poder de explicação. Apesar disso, quando são analisadas cada uma das variáveis independentes individualmente, percebe-se que para algumas delas a hipótese de que não existe relação entre a mesma e o ROA de 2005 não pode ser rejeitada, haja vista que apresenta valor $P$ maior que 5\% (ver Tabela 4), fato que impede a rejeição da hipótese nula para essa variável especificamente. Assim, decidiu-se por excluir tais variáveis do estudo para verificar se seria possível melhorá-lo. O resultado obtido por meio da regressão múltipla está demonstrado na Tabela 8: 


\section{repec}

Flávio Leonel de Carvalho, Andrei Aparecido de Albuquerque, Raphael Pazzetto Gonçalves, Marli Auxiliadora da Silva e Evandro Marcos Saidel Ribeiro

Tabela 8 - Coeficiente de determinação e $F$ de significação do ano de 2004 para previsão de ROE

\begin{tabular}{cc}
\hline & $\mathbf{2 0 0 4}$ \\
\hline$R$-quadrado ajustado & 0,8275 \\
$F$ de significação & 0,0000 \\
Observações & 110 \\
\hline
\end{tabular}

Apesar de retiradas da análise seis das doze variáveis independentes estudadas inicialmente, o poder de ajustamento dos resíduos à reta continuou similar, passando de $83,39 \%$ para $82,75 \%$. O F de significação continuou aproximadamente zero, o que permitiu continuar rejeitando a hipótese nula para o modelo como um todo.

Os pressupostos da regressão também foram testados para essa etapa do trabalho e os resultados são apresentados no Quadro 1.

Quadro 1: Validação da regressão para a previsão de ROA

\begin{tabular}{|c|c|c|}
\hline \multirow{2}{*}{ Teste de Durbin-Watson } & DW & \multirow{2}{*}{ Ausência de Autocorrelação } \\
\cline { 2 - 2 } & 2,0901 & \multirow{2}{*}{ Homocedasticidade } \\
\hline \multirow{2}{*}{ Teste de Pesaran-Pesaran } & valor $p$ & \\
\cline { 2 - 2 } & 0,8787 & \multirow{3}{*}{ Normalidade dos resíduos } \\
\cline { 2 - 2 } Teste de Pesaran-Pesaran & Dteste & \\
\cline { 2 - 2 } & 0,0110 & \\
\cline { 2 - 2 } & Dcrítico & \\
\cline { 2 - 3 } & 0,1297 & \\
\hline
\end{tabular}

Observa-se pelas conclusões do Quadro 1 que os três pressupostos da regressão anteriormente citados foram atendidos. Desse modo, pode-se rejeitar a hipótese nula que afirma que é possível prever o ROA com o uso das variáveis independentes utilizadas no modelo. Logo, existe relacionamento linear entre as variáveis.

Por meio da análise dos valores $p$, rejeitou-se a hipótese nula para todas as variáveis independentes, exceto para a variável $\mathrm{X} 11$, que apresenta valor $P$ acima de $5 \%$, como pode ser observado na Tabela 9.

Tabela 9 - Valor $p$ para as variáveis independentes do ano de 2004 para previsão de ROE

\begin{tabular}{ccc}
\hline & $\mathbf{2 0 0 4}$ & \\
& Coeficientes & Valor $\boldsymbol{p}$ \\
\hline Interseção & $(0,0747)$ & 0,0005 \\
X1 & $(0,1140)$ & 0,0000 \\
X2 & 0,0316 & 0,0000 \\
X3 & 0,6329 & 0,0000
\end{tabular}




\begin{tabular}{ccc} 
X6 & 0,1687 & 0,0014 \\
$X 7$ & 0,1977 & 0,0000 \\
$X 11$ & 0,0875 & 0,2367 \\
\hline
\end{tabular}

Afirma-se, dessa forma, que é possível estimar o ROA com um ano de antecedência, utilizando-se as variáveis independentes com a seguinte ponderação:

$$
R O A=-0,0747-0,114^{*} X_{1}+0,0316^{\star} X_{2}+0,6329^{*} X_{3}+0,1687^{*} X_{6}+0,1977^{*} X_{7}+0,0875^{*} X_{11}
$$

\subsubsection{Previsão do ROA - Etapa 3}

Nessa terceira e última etapa de análise da variância, foi excluída a variável $\mathrm{X}_{11} \mathrm{e}$ todo o procedimento anterior foi repetido. Pela análise do coeficiente de determinação, constatou-se que, com as variações nos indicadores $X_{1}, X_{2}, X_{3}, X_{6}$ e $X_{7}$, é possível a previsão de $82,68 \%$ (Tabela 10) nas variações do ROA. A hipótese nula de que não existe relação entre as variáveis independentes e a dependente é rejeitada, pois, como pode ser observado na Tabela 10, o $F$ de significação do modelo é aproximadamente zero.

Tabela 10 - Coeficiente de determinação e F de significação do ano de 2004 para previsão de ROA

\begin{tabular}{cc}
\hline & $\mathbf{2 0 0 4}$ \\
\hline$R$-quadrado ajustado & 0,8268 \\
$F$ de significação & 0,0000 \\
Observações & 110 \\
\hline
\end{tabular}

No Quadro 2, são apresentados os resultados dos testes para verificação de ausência de autocorrelação, presença de distribuição homocedástica e normalidade dos resíduos. Os três pressupostos da regressão foram encontrados através dos testes de DurbinWatson, Pesaran-Pesaran e Kolmogorov Smirnov.

Quadro 2: Validação da regressão para a previsão de ROA

\begin{tabular}{|c|c|c|}
\hline \multirow{2}{*}{ Teste de Durbin-Watson } & DW & \multirow{2}{*}{ Ausência de Autocorrelação } \\
\cline { 2 - 2 } & 2,1041 & \multirow{2}{*}{ Homocedasticidade } \\
\hline \multirow{2}{*}{ Teste de Pesaran-Pesaran } & Valor P & \multirow{3}{*}{ Normalidade dos resíduos } \\
\cline { 2 - 2 } & 0,8781 & \\
\cline { 2 - 2 } Teste de Kolmogorov-Smirnov & Dteste & \\
\cline { 2 - 2 } & 0,0170 & \\
\cline { 2 - 2 } & Dcrítico & \\
\cline { 2 - 3 } & 0,1297 & \\
\hline
\end{tabular}

Analisando-se individualmente todas as variáveis independentes (Tabela 11), observa-se que as cinco variáveis independentes apresentaram valor $P$ de aproximadamente 


\section{repec}

Flávio Leonel de Carvalho, Andrei Aparecido de Albuquerque, Raphael Pazzetto Gonçalves, Marli Auxiliadora da Silva e Evandro Marcos Saidel Ribeiro

zero, o que reforça a conclusão de que existe relacionamento linear entre as variáveis independentes $\left(\mathrm{X}_{1}, \mathrm{X}_{2}, \mathrm{X}_{3}, \mathrm{X}_{6}\right.$ e $\left.\mathrm{X}_{7}\right)$ de 2004 e o ROA de 2005.

Tabela 11 - Valor p para as variáveis independentes do ano de 2004 para previsão de ROA

\begin{tabular}{ccc}
\hline & $\begin{array}{c}\mathbf{2 0 0 4} \\
\text { Coeficientes }\end{array}$ & Valor $\boldsymbol{p}$ \\
\hline Interseção & $(0,0571)$ & 0,0002 \\
X1 & $(0,1140)$ & 0,0000 \\
X2 & 0,0315 & 0,0000 \\
X3 & 0,6637 & 0,0000 \\
X6 & 0,1603 & 0,0022 \\
X7 & 0,1935 & 0,0000
\end{tabular}

Pela Tabela 11, verifica-se o coeficiente de cada variável independente e, consequentemente, o peso de cada uma delas no modelo preditivo de rentabilidade. Foi obtida a seguinte função preditiva do ROA:

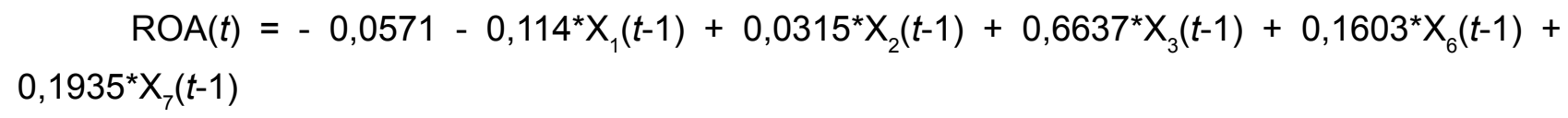

\section{CONSIDERAÇÕES FINAIS}

O objetivo principal deste estudo foi identificar os indicadores contábeis mais significativos para a previsão de rentabilidade das empresas, propondo uma função que, utilizando esses indicadores baseados em dados passados, pudesse prever a rentabilidade futura.

Inicialmente, por meio da técnica estatística de regressão múltipla, verificou-se se os indicadores econômico-financeiros empregados neste estudo poderiam ser utilizados para prever a rentabilidade do Patrimônio Líquido por meio do ROE. Conforme resultados apresentados, não é possivel afirmar que exista relação linear entre os indicadores (variáveis independentes) e o Retorno do Patrimônio Líquido (variável dependente).

Posteriormente, realizou-se o mesmo procedimento com a variável dependente ROA. Apenas as variáveis com um ano de defesagem apresentaram relacionamento linear com um nível de ajustamento do modelo de aproximadamente $83 \%$, com um nível de confiança de $95 \%$. Apesar de esse resultado ser válido para o modelo como um todo, algumas variáveis independentes apresentaram um erro superior a $5 \% \mathrm{e}$, buscando uma melhora do modelo, com um nível de explicação de no mínimo 95\%, retiraram-se essas variáveis da análise repetindo-se o precedimento anteriormente descrito.

Finalmente, pode-se afirmar que as variáveis mais significativas para a previsão de ROA são o Índice de Liquidez $\left(\mathrm{X}_{1}\right)$, Índice de Retenção de Lucros $\left(\mathrm{X}_{2}\right)$, Rentabilidade $\left(\mathrm{X}_{3}\right)$, 
Necessidade de Capital de Giro $\left(X_{6}\right)$ e Saldo em Tesouraria $\left(X_{7}\right)$ com um ano de defasagem em relação ao $R O A$ e com um nível de ajustamento à reta similar ao obtido com a regressão com as 12 variáveis independentes.

\section{CONCLUSÕES}

Os resultados do estudo demonstram que, por meio dos indicadores contábeis, estatisticamente significativos na pesquisa, é possível inferir que a adequada gestão de caixa, além de fundamental para da solvência, é importante para a garantia de rentabilidade futura.

Com base nos resultados encontrados e apresentados, conclui-se que, apesar de existirem polêmicas envolvendo a confiabilidade das informações contábeis, é possível afirmar que os números e os índices financeiros disponíveis nas demonstrações contábeis divulgadas pelas empresas possuem conteúdo informacional que permite a projeção da rentabilidade futura e, consequentemente, a adoção de medidas corretivas quando necessárias.

Conclui-se, ainda, que por meio dos índices contábeis, antecipa-se o conhecimento de futuros problemas financeiros. Assim, com o uso das informações disponíveis nos demonstrativos financeiros, os tomadores de decisão podem adotar medidas com o objetivo de resolver ou mitigar problemas financeiros antes que eles ocorram ou que seus efeitos se tornem mais nocivos à rentabilidade futura das empresas.

\section{REFERÊNCIAS}

ABE, Edson Roberto; FAMA, Rubens. A utilização da duration como instrumento de análise financeira: um estudo exploratório do setor de eletrodoméstico. Caderno de pesquisas em administração, São Paulo, v. 1, n.10, p. 1-12, 3. trim. 1999.

ALTMAN, Edward I. Financial ratios, discriminant analysis and the prediction of corporate bankruptcy, Journal of Finance, v. 23, n. 4, p. 589-609, set. 1968.

ASSAF NETO, Alexandre; SILVA, César Augusto Tibúrcio. Administração do capital de giro. 3. ed. São Paulo: Atlas, 2002.

BEAVER, W. Financial ratios as predictors of failure: empirical search in accounting: selected studies. Journal of Accounting Research, n. 4, p. 71-111, jan. 1966. Suplemento.

CASA NOVA, Silvia Pereira de Castro. Utilização da análise envoltória de dados (DEA) na análise de demonstrações contábeis. 2002. 317 f. Tese (Doutorado em Contabilidade e Controladoria) - Faculdade de Economia, Administração e Contabilidade, Universidade de São Paulo, São Paulo, 2002. 


\section{repec}

CATELLI, Armando (Coord.). Controladoria: uma abordagem de gestão econômica - GECON. 2. ed. São Paulo: Atlas, 2001.

CORRAR, Luiz J.; THEÓPILO, Carlos Renato (coord.). Pesquisa operacional para decisão em contabilidade e administração: contabilometria. São Paulo: Atlas, 2004.

GIMENES, Rogério Marcio Toesca, URIBE-OPAZO, Miguel Angel. Previsão de insolvência de cooperativas agropecuárias por meio de modelos multivariados. Revista FAE, v. 4, n. 3, p. 69-78, set./dez. 2006.

HENDRIKSEN, Eldon S, VAN BREDA, Michel F. Teoria da Contabilidade. Tradução de Antônio Zoratto Sanvicente. São Paulo: Atlas, 1999.

KANITZ, Stephen Charles. Como prever falências. São Paulo: Mcgraw-Hill do Brasil, 1978. Indicadores contábeis e financeiros de previsão de insolvência: a experiência da pequena e média empresa brasileira. 1976. 187 f. Tese (Livre Docência) - Faculdade de Economia, Administração e Contabilidade, Universidade de São Paulo, São Paulo, 1976.

MATARAZZO, Dante Carmine. Análise Financeira de Balanços: abordagem básica e gerencial. 3. ed. São Paulo: Atlas, 1995.

Análise Financeira de Balanços: abordagem básica e gerencial. 5. ed. São Paulo: Atlas, 1998.

SANVICENTE, Antônio Zoratto; MINARDI, Andréa M A Fonseca. Identificação de indicadores contábeis significativos para a previsão de concordata de empresas. Financelab Working Paper - IBMEC, São Paulo, n. 3, p. 01-12, Out. 1998.

SILVA, José Pereira da. Gestão e análise de risco de crédito. São Paulo: Atlas, 1997. 